\title{
A Cost Model for Wavelength allocation Criterion in WDM/TDM PON
}

\author{
Ziyue Gao, Chaoqin Gan, Cheng Fen
}

Key Laboratory of Specialty Fiber Optics and Optical Access Networks, No. 149, Yanchang Road, Zhabei District, 200072 Shanghai, China

Chaoqin Gan: cqgan@shu.edu.cn

Keywords: WDM/TDM-PON; Wavelength Allocation Criterion (WAC); cost model

\begin{abstract}
A cost model is proposed to achieve the wavelength allocation criterion in WDM/TDM PON in this paper. By the cost model, the optimal number of wavelengths allocated can be evaluated in order to be up to the most cost-effective system state. The simulation results demonstrate the correctness of the above conclusion.
\end{abstract}

\section{Introduction}

Hybrid WDM/TDM PON is considered to be the promising evolutionary solution for nextgenerational optical access system. One important issue that needs to be addressed in hybrid WDM/TDM PONs is the wavelength allocation criterion problem [1]. A lot of dynamic wavelength bandwidth allocation (DWBA) algorithms have been proposed to handle this issue [1, 2, 3, 4], but all these studies were considered under the precondition that wavelength allocation is operated with a fixed number of tunable wavelengths. In [5], a joint bandwidth allocation scheme on dedicated and shared wavelength was proposed, which first takes the influence of different number of assigned wavelengths on the network performance into account, but without considering the negative effects introduced by the increment of wavelength number. A wavelength optimization scheme to achieve the balance between performance and cost was proposed in [6], but the relevant performance metric was restricted to queue delay.

In this paper, a cost model is proposed to achieve the wavelength allocation criterion in WDM/TDM PON and its function is to evaluate the optimal number of wavelengths allocated to make sure the system achieve the most cost-effective state.

\section{Network Architecture}

Considering the expansion of construction scale and the integration with the MAN (metropolitan area network), the PON architecture with topology of ring and trees as showed in figure 1 is becoming the trend for large-scale WDM/TDM PON. The ring-tree network is comprised of the optical line terminal (OLT), RNs (Remote Nodes) and ONUs (Optical Network Units). All the RNs are connected to the OLT in the ring topology and the ONUs are attached to each RN.

In this scenario, the OLT can be seen as a central scheduler which collect each ONU's bandwidth-application information before each polling, then, the OLT will allocate wavelengths to each specific RN according to the request bandwidth of ONUs are connected to. The WAC actually provides a most cost-effective wavelength allocated scheme for each $\mathrm{RN}$ to meet the problem of its resource pool assignment. 


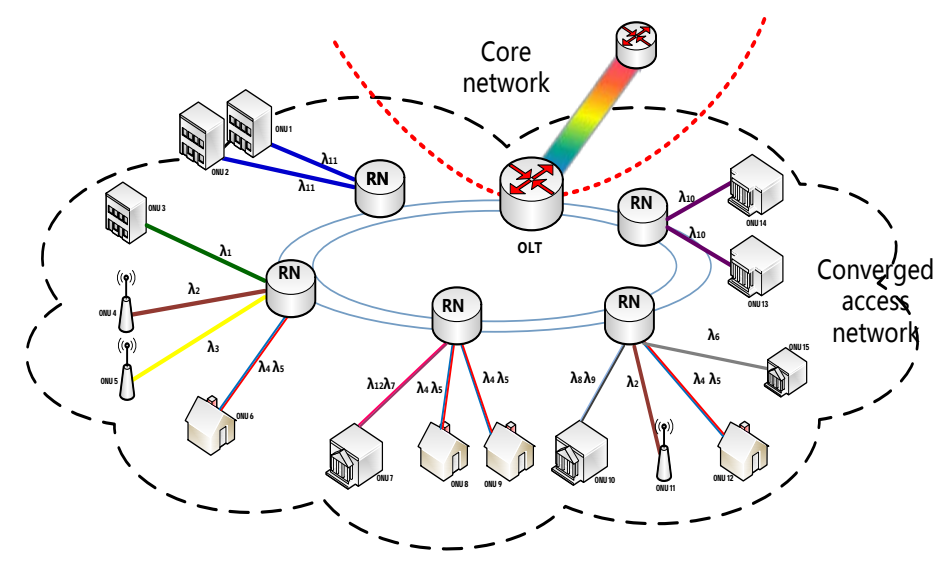

Fig. 1. Network Architecture

To explain clearly, the polling mechanism controlled by REPORT and GATE messages is introduced here to allocate the bandwidth [7]. The first-fit wavelength assignment technique used by the algorithms in [2, 3] are used here to simulate the behavior of the ONUs multiplexing wavelength.

\section{Mathematical Model}

To obtain accurate system cost model, we choose packet dropping probability, packet delay and power consumption as the mainly components of our cost model. In order to acquire the quantitative evaluation of that, we established related mathematical analysis models for each system performance metric mentioned above respectively.

Mathematical Model of Packet Delay. Typical WDM/TDM PON scheduling model with finite buffer at ONUs is shown in Fig. 2.

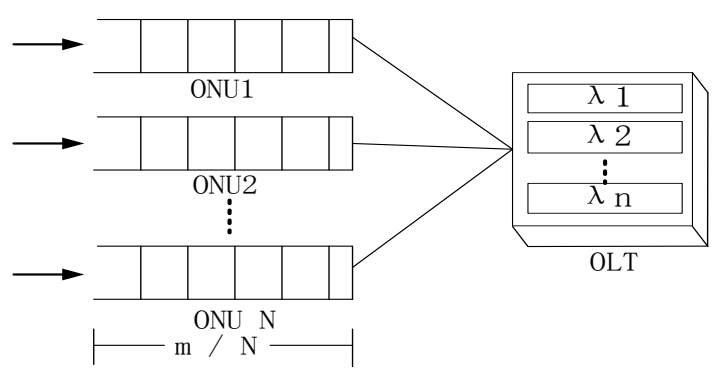

Fig. 2. WDM/TDM PON model with finite buffer

We assume that each ONU's buffer has a maximum capacity with the finite m. Each ONU queue uses a first-in-first-out (FIFO) scheme to select packets for transmission in its TW. To those packets which cannot be assigned wavelengths immediately, they should queue and wait until there are spare wave lengths available. Based the analysis above, we map the wavelength assignment problem into a multiprocessor scheduling problem, and use the $M|M| n \mid$ m model in queuing theory to assist modeling. Combining with the packet delay's modeling idea used in single wavelength circumstances [8]. The total delay can be calculated by

$$
\bar{W}=\overline{W_{F}}+\overline{W_{Q}}+\overline{W_{R}} .
$$

And each of these three parts can be given through the following equation:

$$
\begin{aligned}
& \overline{W_{F}}=c \cdot\left[\rho \cdot \overline{S E_{1}}+(1-\rho) \cdot \overline{S E_{2}}\right] . \\
& \overline{W_{Q}}=c \cdot \bar{W} . \\
& \overline{W_{R}}=\frac{3 N-1}{2 n} \cdot \bar{V} .
\end{aligned}
$$

Where $\mathrm{N}$ denotes the number of ONUs and $\bar{V}$ denotes each ONU's average reservation time (i.e., 
Request packet). The average packet delay for a WDM/TDM PON is

$$
\bar{W}=[\rho \bar{X}+(1-\rho) \bar{V}] \cdot \frac{n^{n} \rho^{n+1} p_{0}\left[1-(\mathrm{m}-\mathrm{n}+1) \rho^{m-n}+(\mathrm{m}-\mathrm{n}) \rho^{m-n+1}\right]}{n !(1-\rho)^{2}\left(\mathrm{n}-\sum_{k=0}^{n-1}(\mathrm{n}-k) p_{k}\right)}+\frac{3 N-1}{2 n(1-c)} \bar{V} .
$$

Mathematical Model of Packet Dropping Probability. For each ONU, when a packet arrives, it is served by a free wavelength based on the first-fit wavelength assignment principle. However, if all the wavelengths are already occupied, then the packet will be queued in the ONU's buffer provided the buffer is not full yet, otherwise, the packet at the tail of the queue will be dropped. This queue management mechanism is known as Drop Tail and it has been most commonly adopted in current optical network, but is also cause problems of Full Queue and Lock-out. In this work, we choose Random Early Detection (RED) as our queue management mechanism to analysis packet dropping probability. The analysis model of packet dropping probability can be established with the use of RED algorithm [9] and queue theory, which calculated as follow:

$$
\begin{aligned}
& P_{b}=P_{\max } \cdot\left(L_{\text {ave }}-n\right) /(m-n) . \\
& L_{\text {ave }}=\sum_{k=n}^{m}(k-n) p_{k}=\frac{n^{n} \rho^{n+1} p_{0}}{n !(1-\rho)^{2}} \cdot\left[1-(m-n+1) \rho^{m-n}+(m-n) \rho^{m-n+1}\right] .
\end{aligned}
$$

$P_{\max }$ indicates the maximum packet dropping probability and $\mathrm{m}$ denote the maximum buffer size, namely, each ONU's buffer size is $m / N$.

Mathematical Model of Power Consumption. The sources of energy waste of shared resource EPON system include: overhearing, control packet over-head, and idle listening [10], by contrast, the major cause of power waste of WDM/TDM PON is wavelength free probability. It means useless energy channels spent on waiting for the traffic to be transmitted. We assume $\bar{n}$ indicate the average number of busy wavelengths, $n$ be the total number of assigned wavelengths, $\eta$ indicate the average channel utilization, $U_{\max }$ indicate the maximum bandwidth utilization of single wavelength, and $e_{p}(k w)$ denote the power consumption by a channel in the OLT. So the mathematical expression of power consumption can be derived below:

$$
\begin{aligned}
& E=\left(1-\eta \cdot U_{\max }\right) \cdot \mathrm{n} \cdot e_{P} . \\
& \bar{n}=\sum_{k=1}^{n-1} k p_{k}+\sum_{k=n}^{m} n p_{k}=n p\left(1-\frac{n^{n} \rho^{m}}{n !} p_{0}\right) . \\
& \eta=\frac{\bar{n}}{n}=p\left(1-\frac{n^{n} \rho^{m}}{n !} p_{0}\right) . \\
& U_{\max }=\frac{R \cdot T_{\text {cycle }}-\frac{N}{n}\left(\mathrm{~S}_{C M}-T_{g} \cdot R\right)-\frac{\left(\mathrm{B}_{D B A}+R T T \cdot R\right)}{n}}{R \cdot T_{\text {cycle }}} .
\end{aligned}
$$

\section{Cost Model}

With the established mathematical model of these system performance metrics, the cost model is given. The management method based on "weight" will be introduced here to establish corresponding system cost model and the optimized function is show as follow:

$$
C=c_{1} \cdot \bar{W}+c_{2} \cdot P_{b}+c_{3} \cdot E .
$$

In the above equation, we use three parameters named $c_{1} c_{2} c_{3}$ to establish a conversion relationship between performance function and cost model. The $\boldsymbol{C}_{\boldsymbol{i}}$ either can be seen as a mark which stand for the corresponding weight in the cost model or can be interpreted into specify cost meaning. Its 
value directly dependent on the certain system performance we are focusing on, for instance, if we focus on power waste, we will assign a large $\boldsymbol{c}_{3}$ to emphasize the affect to energy wasting brought by lower channel utilization. $\boldsymbol{C}_{\boldsymbol{i}}$ also can be used to simulate different variation rules thus it is not necessary to be a constant. For example, the $c_{i}$ of packet dropping probability can be described by the piecewise function below:

$$
c_{2}=\left\{\begin{array}{cc}
c & L_{q} \leq m \\
k \cdot L_{q}+b & L_{q}>m
\end{array} .\right.
$$

When the average queue length less than the maximum limit $m$ specified by RED algorithm, $c_{2}$ is assigned a constant $c$, when the average queue length exceed the $m$, the packet dropping probability comes to 1 , so we can use a linear function related with the $L_{q}$ for expressing $c_{2}$ to increase the cost influence of this part quickly.

\section{Simulation Result}

We study the mechanism of the proposed cost model by conducting a simulation of a hybrid WDM/TDM PON access network model discussed in section II. MATLAB software is used for simulation. We suppose the number of ONUs in each RN is 16, the link capacity is $1 \mathrm{Gbps}$, the mean service for each packet is $5.090 \mu$ s and each ONU has a buffer of $125 \mathrm{Mbytes}$. The total traffic load is $\rho=\lambda / n \cdot \mu$ and $c_{1} c_{2} c_{3}=0.3,0.3,0.4$ respectively, we set the traffic load of one wavelength as the benchmark, then we adjust the arrival rate $\lambda$ of each ONU to make traffic load $\rho$ change, here we use $\rho_{1}=n \cdot \rho$ as the intermediate variable.

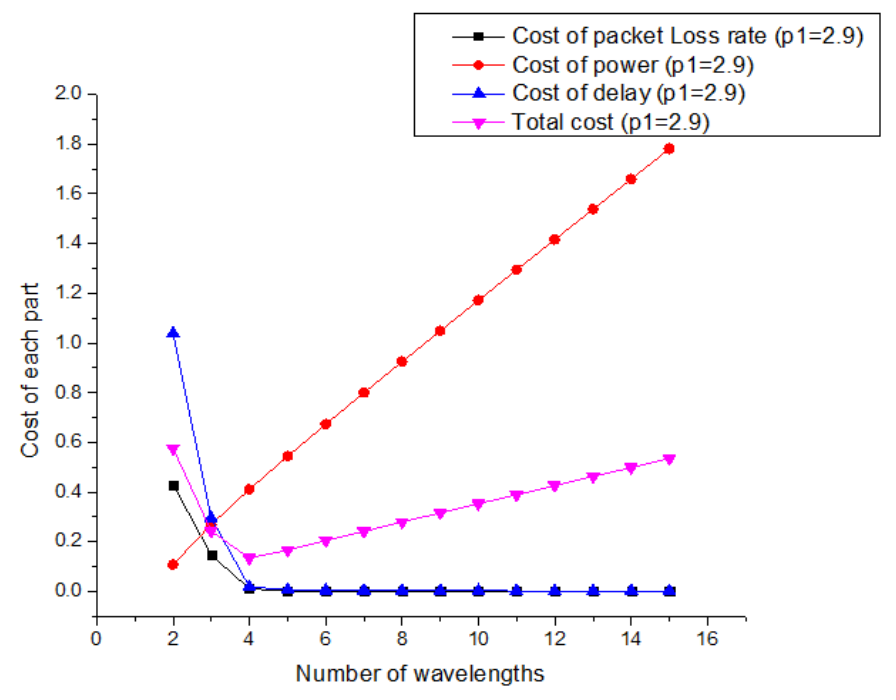

Fig. 3. Cost Curve with Each Components

Fig. 3 shows that the total cost curve and each component of the cost curve, we notice that when the number of wavelengths reached a certain value, though both the delay performance and packet dropping ratio performance still have promotion, but the decisive factor in the total cost has become the cost of power, so it is not necessary to seek to optimize one or two system metrics' performance. 


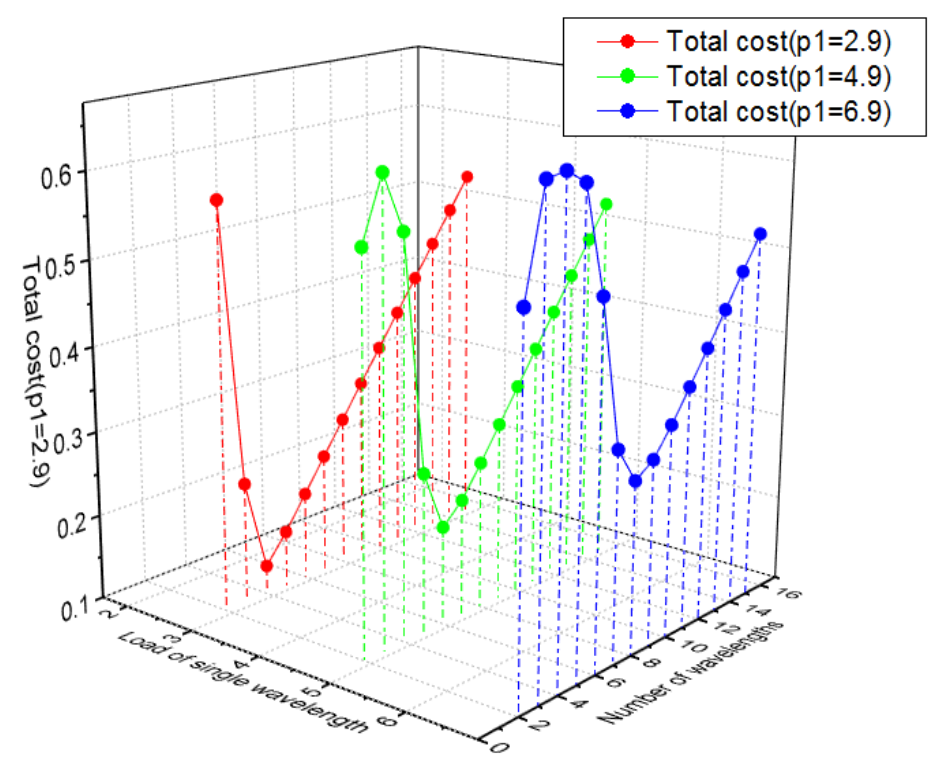

Fig. 4. Cost Curves with Different Traffic Load

The Fig. 4 shows that the optimal number of wavelengths changed from 4 to 6 as the traffic load increase. The decreasing part of each curve is determined by the costs of packet delay and packet dropping ratio, the increasing part of each curve is determined by the cost of power, it is due to the increasing range of the cost of power is more significant than the sum of the cost of packet delay and packet dropping ratio.

\section{Summary}

A cost model is proposed to achieve the wavelength allocation criterion in WDM/TDM PON. It can find out the best number of wavelengths for allocation and achieve the optimal balance between different system performance metrics. The mathematical models of the typical system performance metrics have been derived to establish the cost model. The simulation results indicate that, with the use of the proposed WAC, the optimal wavelength configuration can be achieved, it makes the system most cost-effective.

\section{Acknowledgment}

This work is supported by Programs of Natural Science Foundation of China (No.61132004, 61275073 and 61420106011),Shanghai Science and Technology Development Funds (No. 13JC1402600 and 14511100100), Shanghai Leading Academic Discipline Project (No.S30108), Shanghai Economics and Information Project (No.CXY-2013-93) and Key Laboratory of Specialty Fiber Optics and Optical Access Networks, Shanghai University (No.SKLSFO2012-05).

\section{References}

[1] S.-J. Park, C.-H. Lee, K.-T. Jeong, H.-J. Park, J.-G. Ahn, and K.-H. Song, "Fiber-to-the-home services based on wavelength division-multiplexing passive optical network,” J. Lightwave Technol., vol. 22, no. 11, pp. 2582-2591, Nov. 2004.

[2] Dhaini, A.R., Assi, C.M., Maier, M., Shami, A.: "Dynamic wave-length and bandwidth allocation in hybrid TDM/WDM EPON networks.” IEEE/OSA J. Lightwave Technol.25 (1), 277-286 (2007)

[3] McGarry, M.P. et al.: “Just-in-time scheduling for multichannel EPONs.” IEEE/OSA J. Lightwave Technol. 26 (10), 1204-1216 (2008) 
[4] McGarry, M. P., and Reisslein, M. 2006. “Bandwidth management for WDM EPONs.” Journal of Optical Networking 5(9): 637-654.

[5] Cuiping Ni, Chaoqin Gan, Haibin Chen, "Joint bandwidth allocation on dedicated and shared wavelengths for QoS support in multi-wavelength optical access network," IET Communication. , 2013, Vol. 7, Iss.16, pp. 1863-1870

[6] Benyang Chen, Lei Shi , Chaoqin Gan, "Optimization Model and Simulation Analysis of Wavelength-shared WDM-PON,”

[7] M. P. McGarry, M. Reisslein. “WDM Ethernet Passive Optical Networks,” Communications Magazine, IEEE, 2006; 44 (2): 15 - 22.

[8] F. Aurzadaet al., "Delay analysis of Ethernet passive optical networks with gated service," J. Opt. Netw., vol. 7, no. 1, pp. 25-41, Jan. 2008

[9] Sally Floyd, Van Jacobson, "Random Early Detection Gateways for Congestion Avoidance," IEEE/ACM Transaction on Networking. vol I. no 1. August 199. pp. 397-413

[10] Ying Yan, Lars Dittmann. "Energy Efficiency in Ethernet Passive Optical Networks (EPONs): Protocol Design and Performance Evaluation,” Journal of Communications, vol. 6, no. 3, May 2011, pp.249 